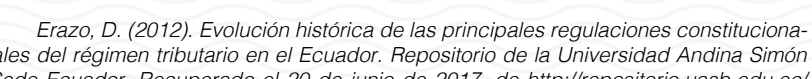

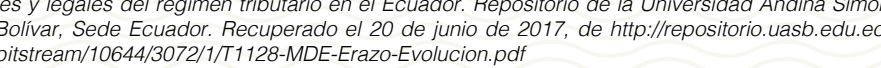

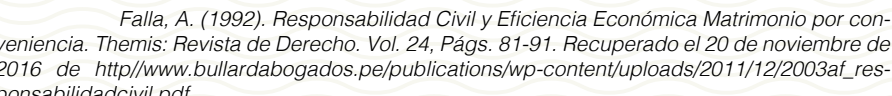

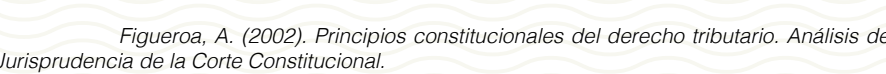

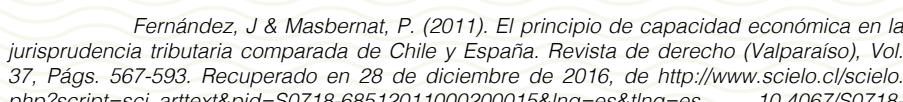
php?script=sci_art

Founrouge, G. (1978). Derecho Financiero. Vol.1. Buenos Aires

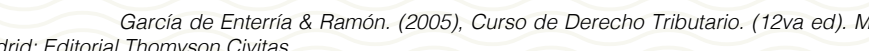
Garcia, R. (1978). Prinoipiois de Derecho Tributario. Buenos Aires: Ediciones

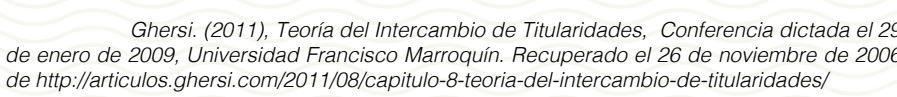
Gimini A (1980) no

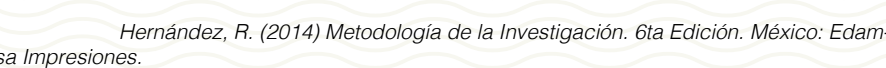

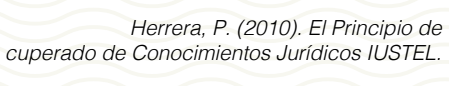

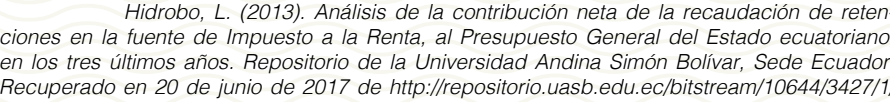

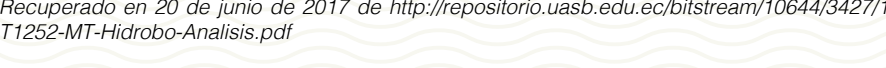
Nacional, Loja:

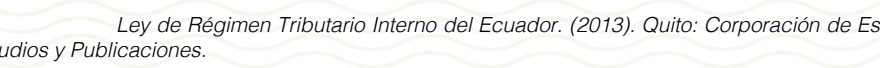

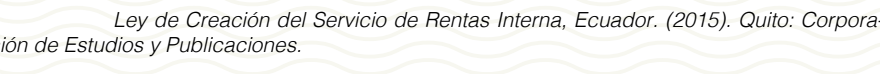

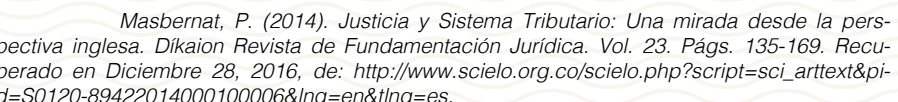

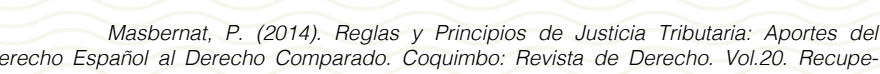

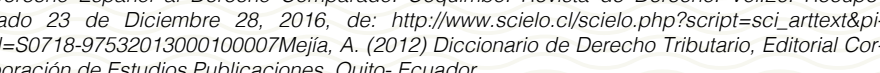

Michell, G. (1975). Curso de Derecho Tributario. Caracas: Editoriales de Derecho

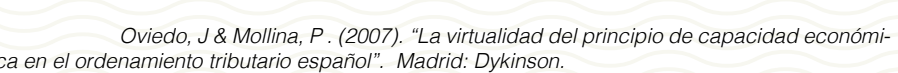

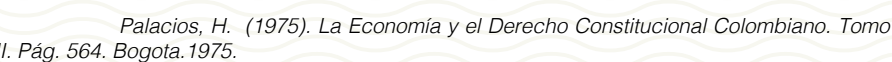

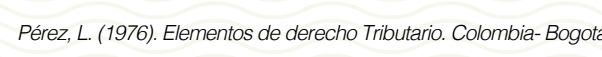
Podetti, R. (1969). Tratado de los recursos. Pág. 126.

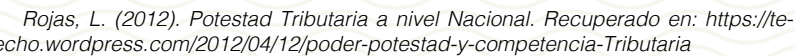

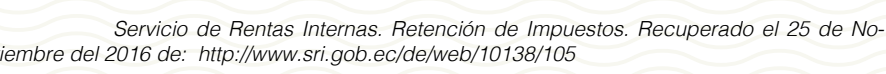

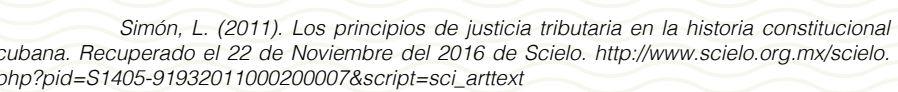

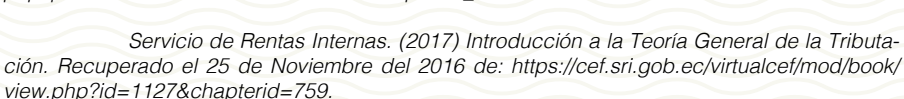

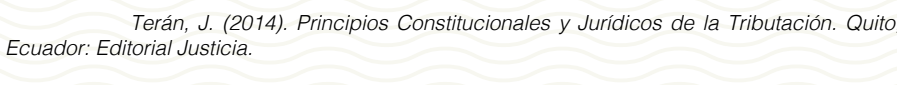

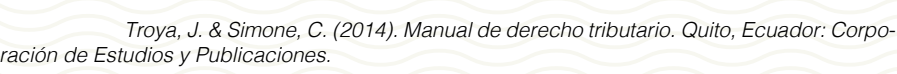

\title{
Proyectos de vivienda rural con bono habitacional en Tungurahua
}

\section{Fernando Mayorga-Núñez ${ }^{1}$ Licett Freire-Paredes ${ }^{2}$ Tatiana Vayas-Carrillo ${ }^{3}$}

Fecha de recepción: 16 de Junio 2017

Eesumen

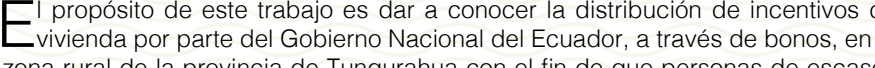
recursos económicos puedan acceder a una vivienda digna que mejore su calida

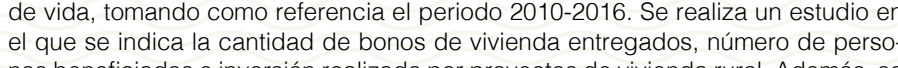

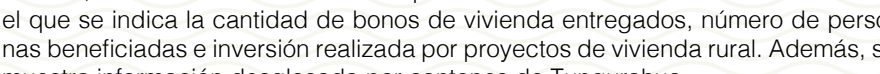
Palabras clave: Bono de vivienda, calidad de vida, incentivo, inversión, proyecto
devivienda rural Thstract purposen

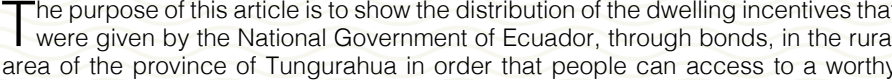
dwelling that improves their quality of life, taking as reference the period 2010
2016. This study shows the number of dwelling bonds given to people the 2 numb

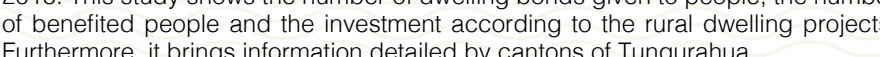
(a) Introducción
La vivienda

Lavivinda es un derecho humano fundamental que influye directamente en

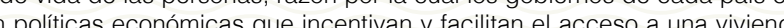

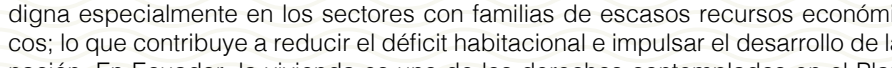

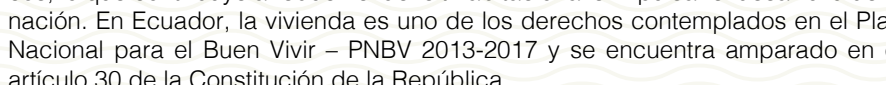

Según el Banco Interamericano de Desarrollo - BID (2012), una de cada tres fant
lias de America Latina y el Caribe $(59$ millones de personas) habitita en una vivient da inadecuada o construida con materiales inseguros o que no posee servicios
basicos; y más del $50 \%$ de familias en 41 ciudades principales latinoamericanas carecen de medios para comprar una vivienda adecuad

En el pais han existido tres politicas de vivienda, la primera nacida en los ańn
veinte dentro del entorno municipal y la seguridad social que consistia en "progra mas o proyectos residenciales". Luego, a partir de los años sesenta se implementi la producción y promoción de viviendas cuyo sistema de financiamiento se hasa

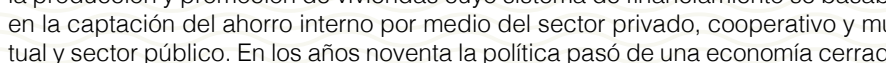

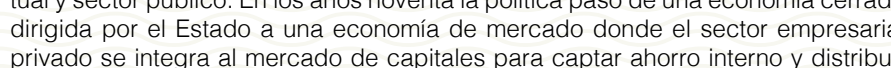

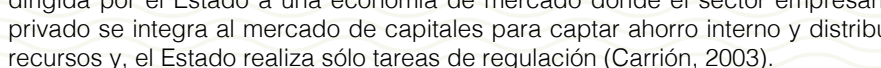
Durante los últimos años se ha implementado el Sistema de Incentivos para
Viviend a - SIV anivel nacional, el cual consiste en un coniunto de bionos dirigidos a la ciudadania, entregado por el Gobierno Nacional a traves del Ministerio de

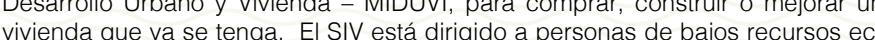

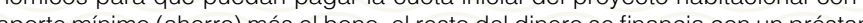
hipotecario otorgado por el Banco del Instituto Ecuatoriano de Seguridad Soc a - BIESS, o a su vez por medio de la banca privada u otras mutualistas aliadas a
Gobierno Nacional. II SIV pretende reduciri el defíitit de vivienda que, seguin e

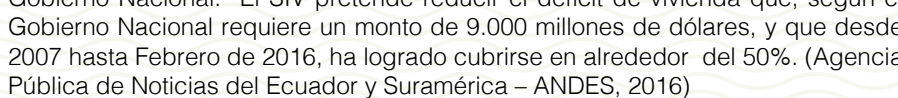

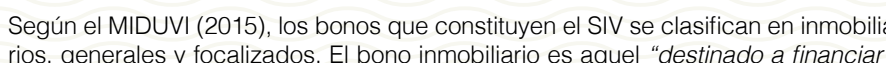

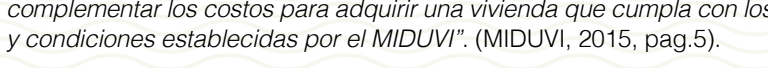

Fecha de aceptación: 30 de Junio 2017

EI MIDUVI (2015) indica que el bono general puede ser

- Bono para construcción de vivienda nueva en terreno propio sea urbana, rura
- amazónica el cual sive para financiar o complementar la construcción de una vivienda sobre el terreno del beneficiario en los siguientes casos: cuando no exista no recuperable. (..6)

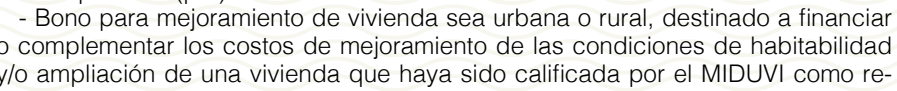
Ylo ampliación de una vivienda que haya sido calificada por el MIDUVI como re-
cuperable. (p.6)

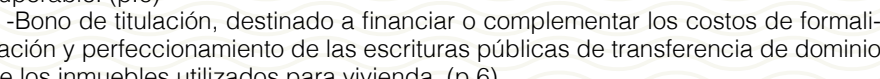

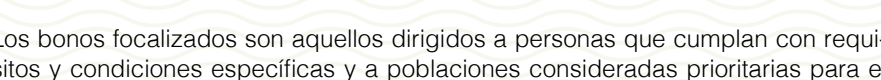
pais en la lucha de erradicación de la pobreza; dentro de estos bonos se encuen. Las metas a alcanzar en la lucha de la erradicación de la pobreza sone erradica

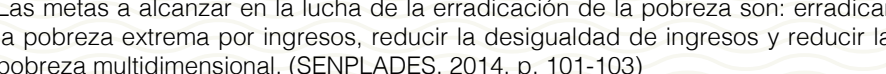

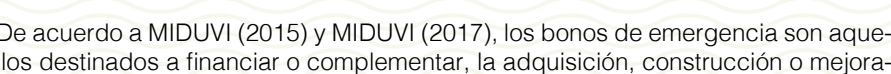
miento de una vivienda que ha sido o puede ser afectada por desastres generados azones humanitarias para la atención de grupos poblacionas des de emergenciaia por

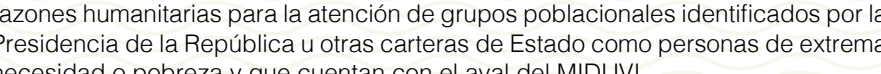

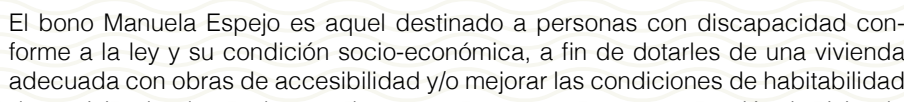
de su vivienda, dentró de estos bonos tenemost tres: para construcción de vivienda nueva con adquisicíón de terreno, para construcción de vivienda nueva en terreno

$$
\begin{aligned}
& \text { Tabla 1. Valores de bonos de vivienda en Ecuador } \\
& \text { 2015-2017 }
\end{aligned}
$$

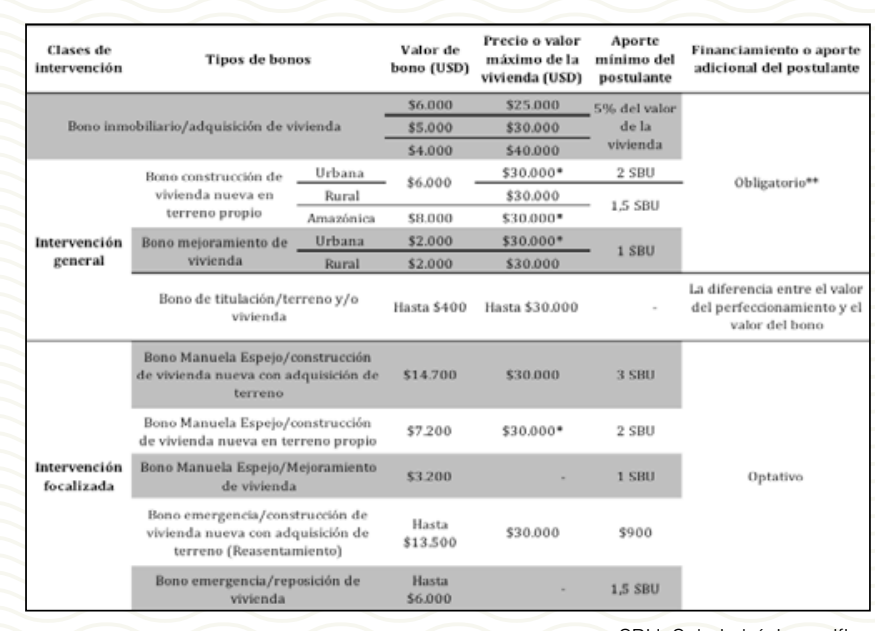

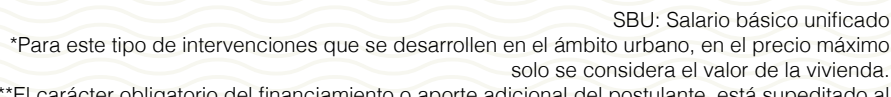

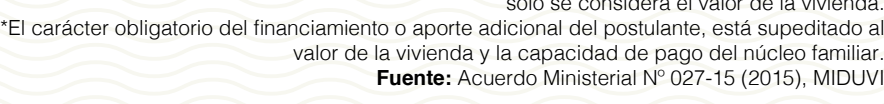


Bajo el contexto mencionado, se considera importante dar a conocer el desarrollo
del SIV en el área rural de Tungurahahua, puesto que contribuye como instrumento para el mejoramiento de pollticas públicas en esta aárea y, además, el planteamien-

Metodología
Se realizi una
De

Se realizo una investigación descriptiva, utilizando intormacion sobre proyectos
de vivienda rural con bono habitacional, periodo $2010-2016$, otorgados por el $M$.

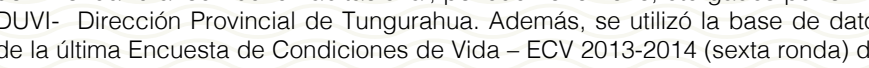
Institutu Nacional de Estadisitica y Censos- INEC y se usaron tórmulas estadisitica

Resultados

Sector rural Tunguranua
De acuerdo a $a$ ECV $2013-2014$

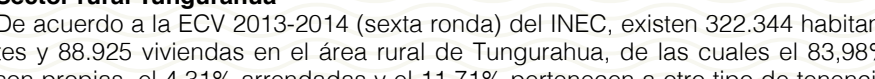

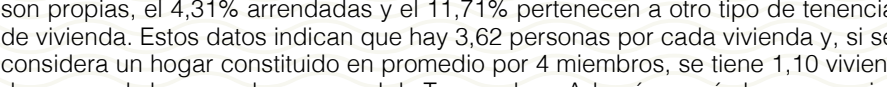

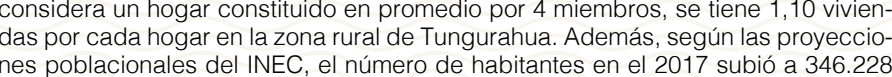
nes poblacionales del
(incremento del $7.41 \%$ ).

Entre 2010 y 2016 , el SIV en Tungurahua se desemperín en varios proyectos
Vivienda rural los cuales, a manera de resumen, abarcan cuatro categorias: lervención vivienda rural, intervención focalizada - Manuela Espejo, intervenciion 2809 bonos de vivienda con una inversión de $\$ 200^{\circ} 526.140,34$ en beneficic 11.236 habitiantes. La mayor actividad se registrś en 2010 donde se otorgare do a 4.312 individuos.

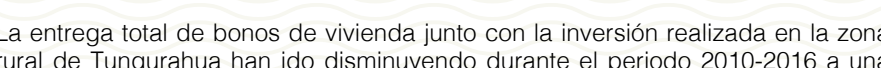

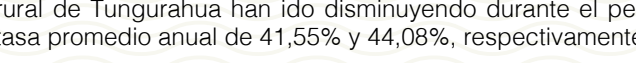

Tabla 2 . Proyectos de viviendar ural en Tungurahua

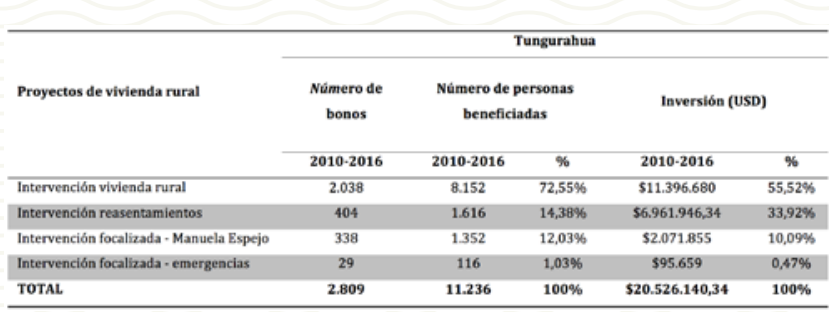

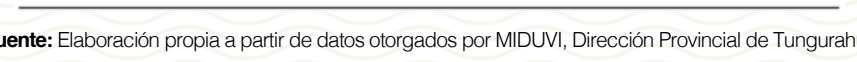

Al desglosar el t total de bonos de vivienda por cantones de Tungurahua, Pellieo po-
see el mayor porcentaie de bonos recibididos $(30.05 \%)$, seguido por Quero (17,76\%

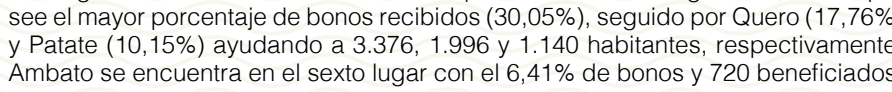

$$
\text { Tabla 3. Bonos de vivienda urual por cantones de Tungurahua }
$$

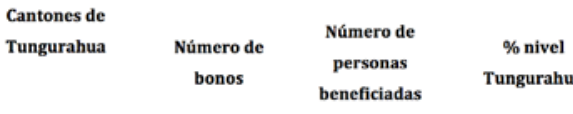

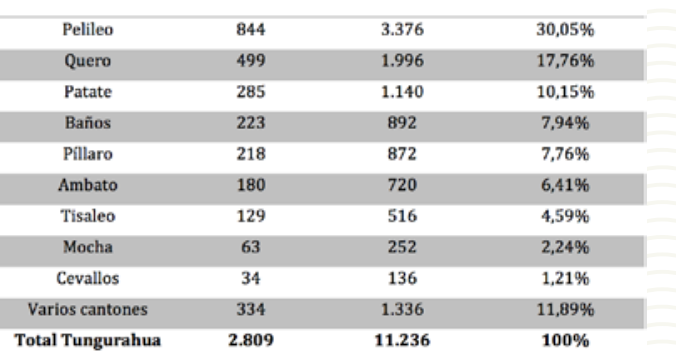

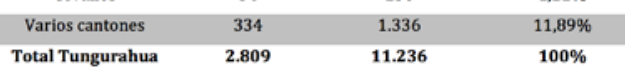

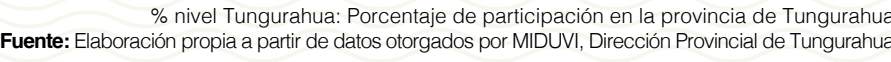

Del 2010 al 2016 , los proyectos de intervención vivienda rural en Tungurahua han
obtenido el mayor número de bonos con un total de 2.038 en beneficicio de 8.152

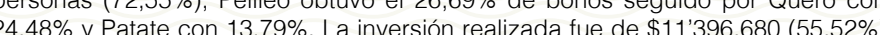
sendo el año 2010 el de mayor desembolso con $\$ 3278.000$ (15,97\%) correspon-

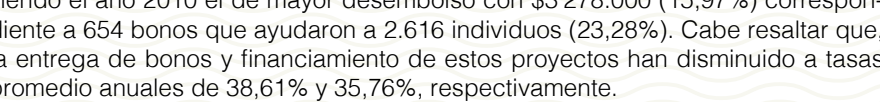

Tabla 4. Bonos de intervención vivienda rural por cantiones de Tungurahua
$2010-2016$ Interenencion viviendar rut
$2010-2016$
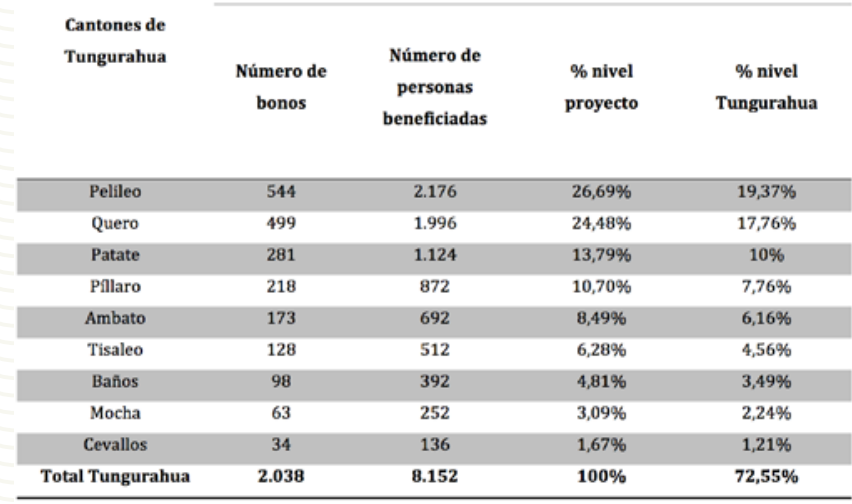

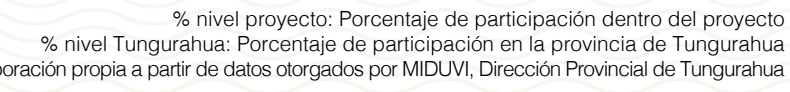
Los proyectos de intervencíín reasentamientos en Tungurahua, se desarrollaron
en los años 2010 y 2012 , con un total de 404 bonos (14,38\%), Los cantones que reistrtran estos proyectos son: Pelilieo con el $69,31 \%$ y Baños con 30,69\% en bonos.

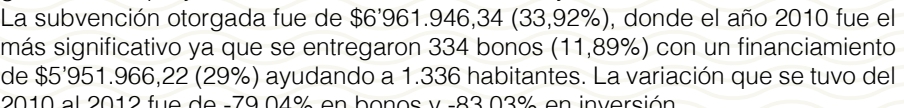
Tabla 5. Bonos de intevenención reasentamientios por cantiones Tunguranua

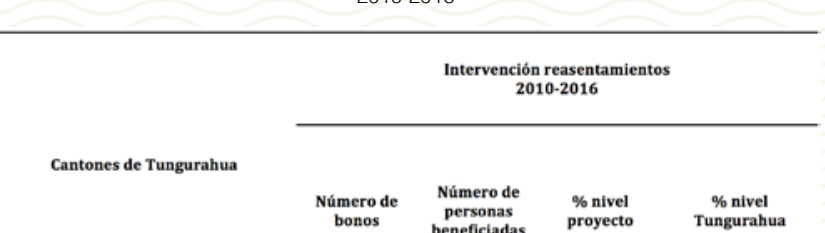
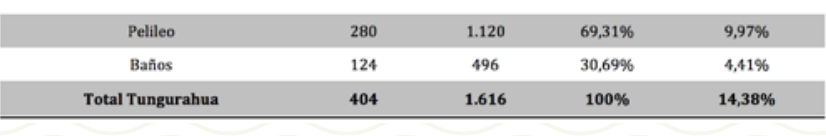

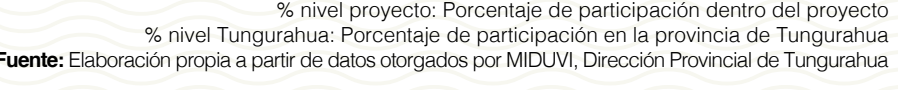
En referencia a los bonos de vivienda para proyectos de intervención - Manuela
Espejo, se entregaron 338 con un desembolso de $\$ 22071.855(10,09 \%)$ y 1.352

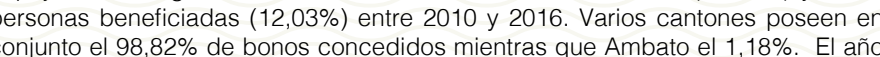
de mayor actividad fue el 2012 con $132 \mathrm{bonos}(4,70 \%$ ) que ayudaron a 528 individe dinero invertido en estos provectos han decrecido a tasas promedio anualies de

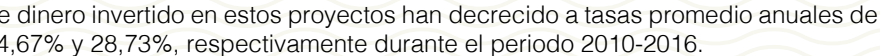

\begin{tabular}{|c|c|c|c|c|}
\hline & & 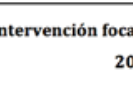 & 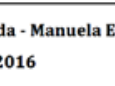 & \\
\hline ranhas & $\begin{array}{c}\text { Nímero de } \\
\text { bonos }\end{array}$ & 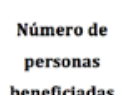 & $\begin{array}{l}\text { \% nivel } \\
\text { provecto }\end{array}$ & $\begin{array}{c}\text { \% nivel } \\
\text { Tungurah }\end{array}$ \\
\hline
\end{tabular}

$\underset{\substack{\text { Ambato } \\ \text { Varios cantone }}}{\cos }$

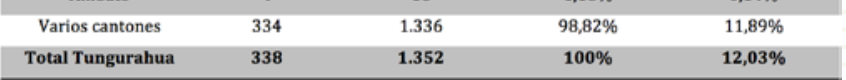

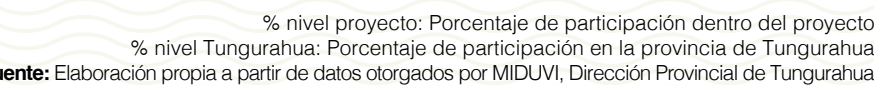
Con respecto a intervencion focalizada - emergencias, se totragaron 29 bonos canton que registra el mayor porcentaje de bonos (68.97\%) seguido por Patal siendo 2011 el año de mayor subvención con $\$ 55.800(0,27 \%)$ pero, el añó 2012 fue el de mayor porcentiaje de bonos $(0,71 \%)$. Los bonos e inversiones de estos proyectos se han reducido a a
pectivamente entre 2011 y 2016 .

Tabla 7. B Bonos de intervención focalizada- - mergencias, por cantones Tungurahua
$2011-2016$

$$
\begin{aligned}
& \begin{array}{l}
\text { Intervencion focalizada- energencias } \\
2011-2016
\end{array} \\
& \text { Tungurahus }
\end{aligned}
$$

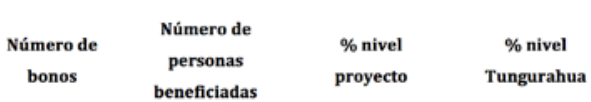

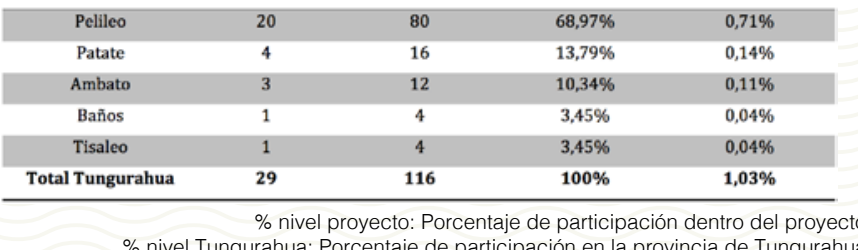

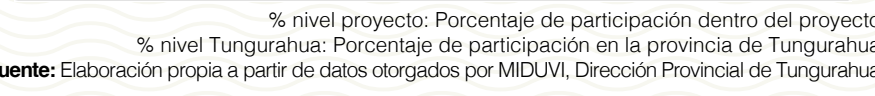

N Tungurathua se entregaron 2.809 bonos de vivienda rural con una inversión d
$20526.140,34$ en beneficicio de 11.236 personas del 2010 al 2016 . Durante los

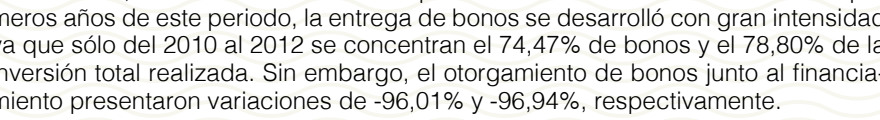

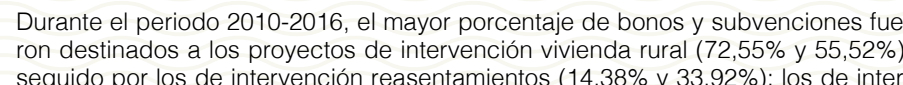

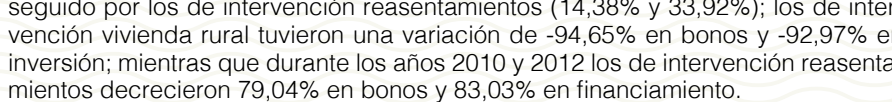

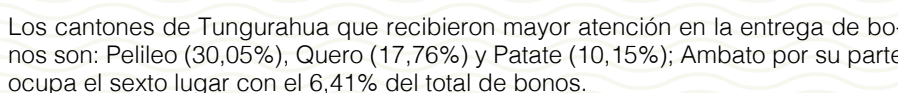

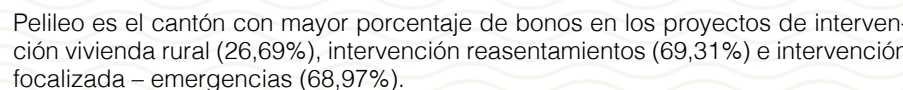
Los resultados de esta invessigación constituyen una herramienta para la toma de
decisioiones por parte de las Instituciones del Estado Ecuatoriano encarganadas de

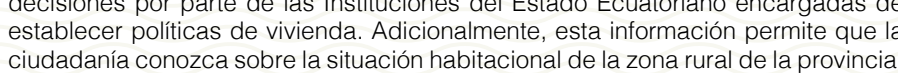

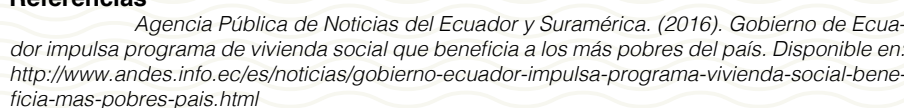

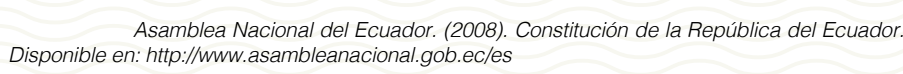

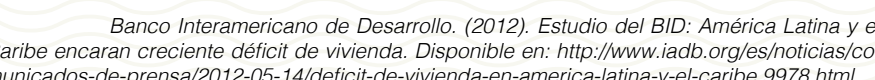

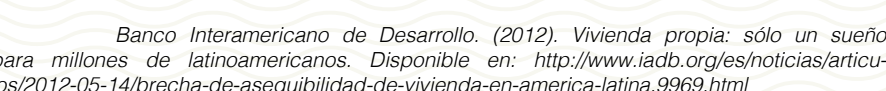
Carrión, F. (18 de enero de 2003). El problema de la v vivenda en el Ecuador. Diarto

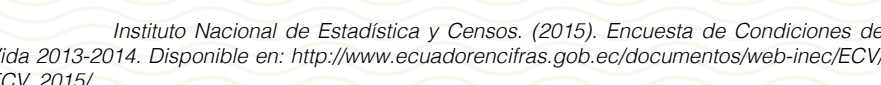

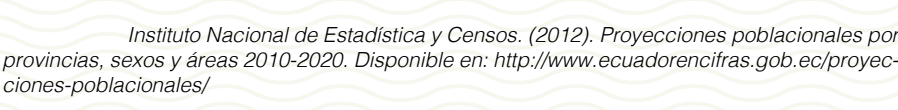

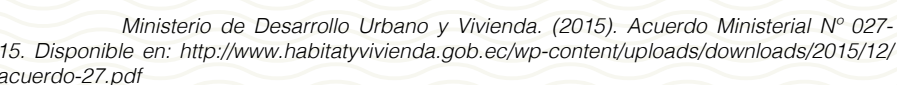

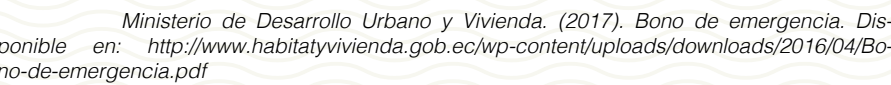

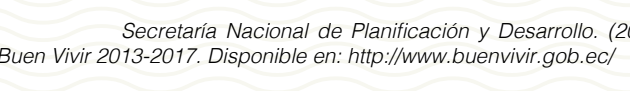

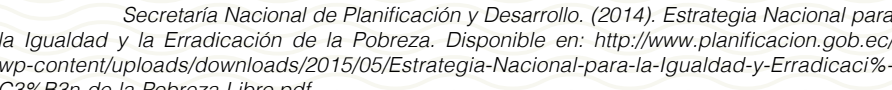

Proyectos de vivienda rural con bono habitacional en Tungurahua 